\title{
Lymphoid and epithelial markers in small cell anaplastic thyroid tumours
}

\author{
AD BURT, ${ }^{*}$ DJ KERR,${ }^{* *}$ IL BROWN, ${ }^{*}$ P BOYLE ${ }^{* * *}$ \\ From the ${ }^{*}$ University Department of Pathology, Western Infirmary, Glasgow, the ${ }^{* *}$ Cancer Research \\ Campaign University Department of Oncology, Glasgow, and the ***West of Scotland Cancer Surveillance \\ Unit, Ruchill Hospital, Glasgow
}

SUMMARY The expression of the lymphoid marker, common leucocyte antigen and of the epithelial marker, epithelial membrane antigen by small cell anaplastic thyroid tumours was studied in 53 tumours, using the peroxidase-antiperoxidase and avidin-biotin techniques. Common leucocyte antigen was found in 33 of the 53 tumours; six tumours were positive for epithelial membrane antigen; and the remaining 14 tumours were negative for both markers. These results support the suggestion that most small cell anaplastic thyroid tumours are of lymphoid origin. Survival data for patients with tumours positive for common leucocyte antigen showed a significantly better prognosis than did the data for patients with small cell tumours that did not express this marker $(\mathrm{p}<0.02)$.

Small cell anaplastic tumours account for $15 \%$ of all thyroid malignancies.' The histogenesis of such tumours has been the subject of considerable debate, ${ }^{2}$ but they are now considered to be of lymphoid or epithelial origin, ${ }^{3}$ lymphomas being the more common. ${ }^{34}$ It is often impossible for the histopathologist, however, to distinguish between the two groups on morphological grounds alone. Histological criteria for this distinction have been outlined ${ }^{5}$ but are often difficult to apply, particularly when specimens obtained by needle biopsy are being dealt with.

The clinical importance of distinguishing between lymphoma and anaplastic carcinoma remains uncertain. It has been suggested that lymphomas respond more favourably to radiotherapy and have a better prognosis than anaplastic carcinomas. ${ }^{6}$ Much of the evidence for this is anecdotal, however, and in a large retrospective study Rossi et al found no significant difference in survival between the two groups. ${ }^{7}$ In their study Rossi and his colleagues relied on the use of histological criteria alone for distinguishing between the two tumour types.

The purpose of this study was to attempt to distinguish between thyroid lymphomas and small cell anaplastic carcinomas by using the immunohistochemical finding of lymphoid and epithelial cell

Accepted for publication 1 May 1985 markers. Furthermore, using these results, we assessed the correlation between presumed histogenesis and survival.

\section{Material and methods}

Material, fixed in formalin and embedded in paraffin, from 10 well differentiated thyroid carcinomas (five papillary, five follicular) and 53 small cell anaplastic thyroid tumours was obtained from the files of the department of pathology, Western Infirmary, Glasgow. Tumours in which spindle cells or giant cells were present were not included in the study. Of the 53 anaplastic tumours, 21 had been histologically regarded as lymphomas, 13 as anaplastic carcinomas, and 19 as anaplastic tumours of uncertain histogenesis. Retrospective clinical data were available for 40 patients in the group with the anaplastic tumours.

Sections $5 \mu \mathrm{m}$ thick were used for the immunohistochemical studies. The primary antibodies used were anticommon leucocyte antigen (mouse monoclonal; Dako), antiepithelial membrane antigen (rabbit polyclonal; Sera-Lab), and antithyroglobulin (rabbit polyclonal; Dako). Common leucocyte antigen was shown using the avidin-biotin technique, ${ }^{8}$ and epithelial membrane antigen and thyroglobulin were shown using the peroxidase-antiperoxidase technique.' Sections were counterstained with haematoxylin and viewed under a Leitz interference contrast microscope. 


\section{Results}

WELL DIFFERENTIATED THYROID CARCINOMA Nine out of 10 well differentiated thyroid carcinomas showed strong positive staining for epithelial membrane antigen, with weak focal positivity in the remaining case. This antigen was distributed in a pattern similar to that seen in normal thyroid tissue, with maximal staining on the cell membrane along the luminal border. All 10 tumours showed positive staining for thyroglobulin. None was positive for common leucocyte antigen.

\section{ANAPLASTIC TUMOURS}

Six of the 53 anaplastic tumours were positive for epithelial membrane antigen. This was predominantly distributed in bound linear membranes (Fig. 1), though in three cases sporadic tumour cells showed diffuse intracytoplasmic staining. Thyroglobulin was not detected in the anaplastic tumours. Thirty three tumours were positive for common leucocyte antigen. Staining for this antigen was exclusively linear membrane bound (Fig. 2). No tumour was found to express both common leucocyte antigen and epithelial membrane antigen.

\section{COMPARISON BETWEEN HISTOLOGICAL DIAGNOSIS AND IMMUNOHISTOCHEMICAL}

RESULTS

Nineteen out of 21 tumours initially regarded as lymphomas were positive for common leucocyte antigen. One tumour was positive for epithelial

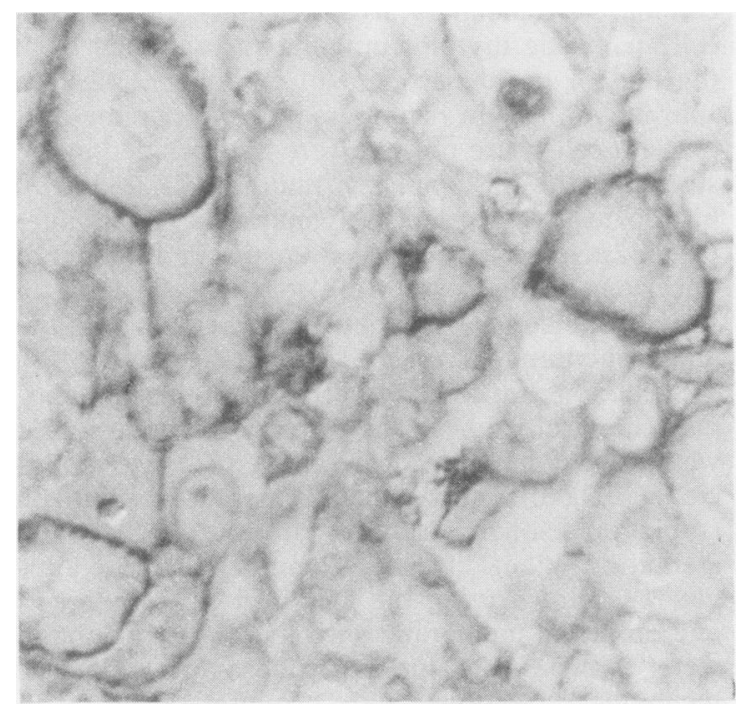

Fig. 1 Membrane staining for epithelial membrane antigen. (Peroxidase-antiperoxidase.) $\times 520$.

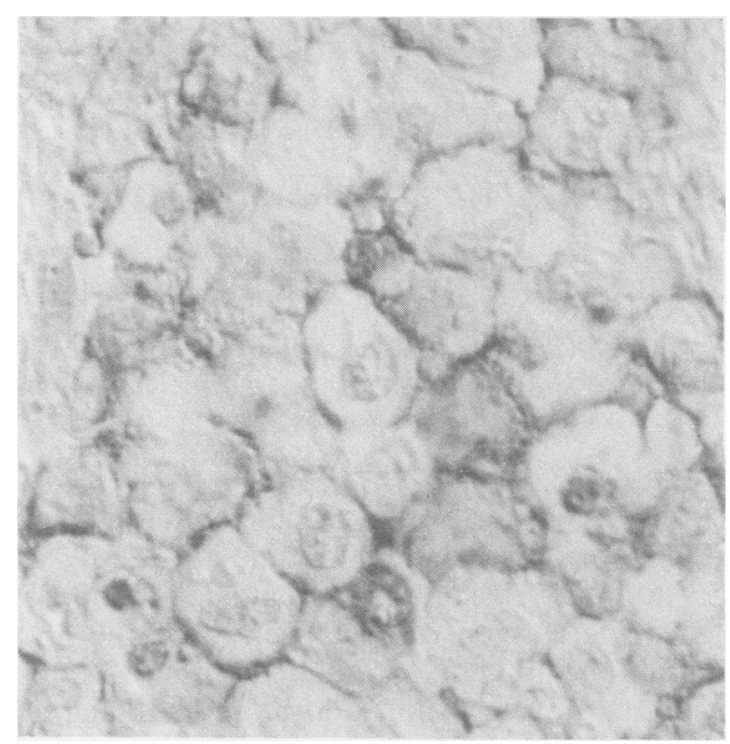

Fig. 2 Membrane staining for common leucocyte antigen. (Avidin-biotin.) $\times 450$.

membrane antigen and was reclassified as an anaplastic carcinoma. Three of the tumours initially regarded as anaplastic carcinomas were positive for common leucocyte antigen and were reclassified as lymphomas. In 12 out of 19 tumours previously diagnosed as anaplastic tumours of uncertain histogenesis, 11 were positive for common leucocyte antigen and one positive for epithelial membrane antigen: these were reclassified as lymphomas and carcinoma, respectively. Fourteen tumours that were negative for both markers remained unclassifiable.

\section{CLINICAL DATA}

Well documented clinical follow up was available in 40 cases. Of these cases, 26 were positive for common leucocyte antigen, four were positive for epithelial membrane antigen, and 10 were negative for both markers. There was no significant difference between these three groups with respect to mean age, presenting signs and symptoms, tumour stage at presentation, or method of treatment. There was a preponderance of women of 10:1 in the group positive for common leucocyte antigen and $4: 1$ in the group negative for common leucocyte antigen and epithelial membrane antigen. All of the patients positive for epithelial membrane antigen were women. The median survival of patients with tumours positive for common leucocyte antigen was 13 months, of those with tumours positive for epithelial membrane antigen 3.5 months, and of those with tumours negative for common leucocyte 


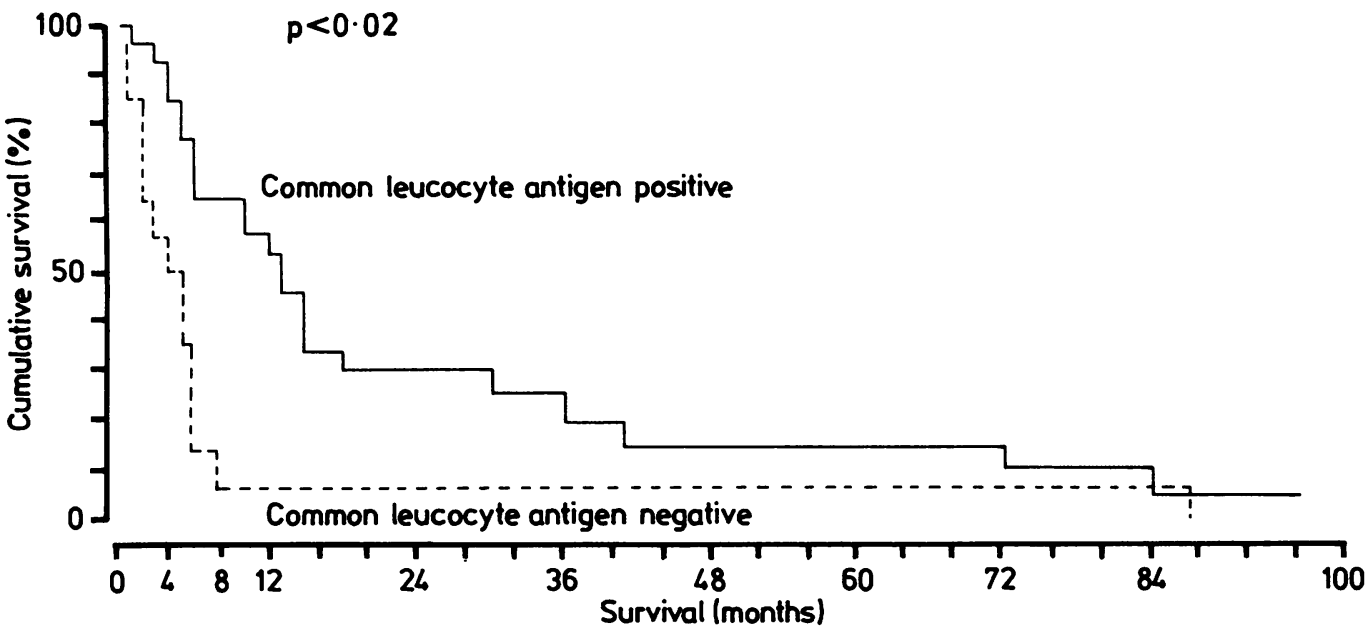

Fig. 3 Kaplan-Meier survival curves for patients with tumours positive and negative for common leucocyte antigen.

antigen and epithelial membrane antigen five months.

Kaplan Meier survival curves for patients with tumours positive for common leucocyte antigen were compared with those for patients with tumours negative for common leucocyte antigen (Fig. 3). The group with tumours negative for common leucocyte antigen therefore included patients with epithelial membrane antigen positive tumours and common leucocyte antigen/epithelial membrane antigen negative tumours. Comparison of the two groups showed a significantly better survival for patients with common leucocyte antigen positive tumours $(\mathrm{p}<0.02)$.

\section{Discussion}

Small cell anaplastic tumours of the thyroid commonly present diagnostic difficulties for the histopathologist. In routine histological preparations these tumours are characterised by the presence of small malignant cells growing diffusely and compactly with little evidence of differentiation. In previous reports some of these tumours were regarded as sarcomas, ${ }^{2}$ but it is now thought that most small cell neoplasms of the thyroid are either lymphomas or small cell anaplastic carcinomas. Differentiation between these two types on purely morphological grounds is often impossible; electron microscopy may help $p^{10}$ but may introduce sampling problems. Using immunohistochemical methods in a series of 10 small cell tumours, Mambo et al attempted to establish the histogenesis using thyroxine and immunoglobulin light chains as their respective epithelial and lymphoid markers. ${ }^{3}$ Six tumours were identified as lymphoid and two as epithelial, although the other two remained unclassifiable. The use of light chains as lymphoid markers, however, is likely to underestimate the true incidence of lymphoid tumours in that only B cell neoplasms will be detected.

In this study we used common leucocyte antigen as a lymphoid cell marker and epithelial membrane antigen as an epithelial cell marker. Such a combination has been used in the differential diagnosis of large cell anaplastic tumours." Common leucocyte antigen is a cell membrane protein that is present on all leucocytes and well differentiated lymphomas. ${ }^{12}$ Thirty three tumours in our study (62\%) expressed this antigen, thus supporting the theory that most small cell thyroid tumours are lymphomas. ${ }^{4}$ Epithelial membrane antigen is also a membrane bound antigen, which is expressed on a wide range of epithelial cells and also on mesothelial cells. ${ }^{13}$ In their study Sloane et al showed that epithelial membrane antigen was expressed by well differentiated thyroid carcinomas. ${ }^{13}$ In our study we confirmed this, finding strong staining for this antigen in nine out of 10 tumours; however, epithelial membrane antigen was found in only six anaplastic tumours. None of these tumours coexpressed common leucocyte antigen, and we consider that they may therefore be regarded as probable epithelial tumours. Delsol et al recently questioned the specificity of epithelial membrane antigen for epithelial cells and confirmed its presence on plasma cells. ${ }^{14}$ These cells, however, would be expected to coexpress common leucocyte antigen, and any tumour that exclusively expressed epithelial membrane antigen might be considered to be of probable epithelial origin. The 
lack of expression of thyroglobulin by the anaplastic tumours affirms the result of previous studies. ${ }^{15}$

On the basis of our immunohistochemical results, therefore, we reclassified $16(30 \%)$ of our retrospective series of anaplastic small cell carcinomas. The ease with which the immunohistochemical technique can be applied on material embedded in paraffin means that antibodies to common leucocyte antigen and epithelial membrane antigen should be useful diagnostic tools in the investigation of anaplastic thyroid tumours. There remains a group of tumours, however, that express neither epithelial membrane antigen nor common leucocyte antigen. The histogenesis of these undifferentiated tumours remains uncertain.

It has often been suggested that thyroid lymphomas respond more readily to radiotherapy and have a better prognosis than small cell carcinomas. ${ }^{410}$ There have been few studies in which survival data for the two groups have been compared: Rossi et al studied 147 anaplastic thyroid tumours and divided these into small cell compact carcinomas, small cell diffuse carcinomas, and lymphomas on the basis of their histological appearances. ${ }^{7}$ One and five year survival figures were used in the analysis of the data. They showed no significant difference in survival between the patients with small cell diffuse carcinoma and those with lymphoma, though the patients with small cell compact carcinoma showed a poorer five year survival. These results must be interpreted with caution, given the accepted limitations of histological criteria in distinguishing between lymphomas and carcinomas.

In this study we compared survival curves for patients with common leucocyte antigen positive tumours with those for patients with common leucocyte antigen negative tumours using Kaplan-Meier statistical analysis. We found a significant difference in survival between the two groups. These results support the suggestion that presumed lymphoid tumours carry a more favourable prognosis than small cell carcinomas. The immunohistochemical proof of common leucocyte antigen in anaplastic thyroid tumours may therefore provide information that could be of prognostic importance. Furthermore, with the development of chemotherapeutic regimens specific for different types of tumour information relevant to the management of patients with such tumours may also result.
We thank Mr J Stewart for technical help and Miss E Sharkie for typing the manuscript. The study was supported in part by a grant from the Scottish Home and Health Department and by the research fund of the faculty of medicine, University of Glasgow.

\section{References}

' Meissner WA, Warren S. Tumours of the thyroid gland. In: Meissner WA, Warren S, eds. Atlas of tumour pathology. 2nd series, fascicle 4. Washington DC: Armed Forces Institute of Pathology, 1969.

${ }^{2}$ Winship T, Greene R. Reticulum cell sarcoma of the thyroid gland. Br J Cancer 1955;9:401-6.

${ }^{3}$ Mambo NC, Irwin SM. Anaplastic small cell neoplasms of the thyroid: an immunoperoxidase study. Hum Pathol 1984; 15: 55-60.

${ }^{4}$ Compagno J, Oertel JE. Malignant lymphoma and other lymphoproliferature disorders of the thyroid gland: a clinicopathologic study of 245 cases. Am J Clin Pathol 1980; 74: 1-11.

5 Meissner WA, Philips MJ. Diffuse small cell carcinoma of the thyroid. Archives of Pathology 1962;74:291.

- Rayfield EJ, Nishiyama RH, Sisson JC. Small cell tumour of the thyroid. Cancer 1971;28:1023-30.

' Rossi R, Cady B, Meissner WA, Sedgwick CE, Werber J. Prognosis of undifferentiated carcinoma and lymphoma of the thyroid. Am J Surg 1978;135: 589-95.

${ }^{8}$ Hsu SM, Raine L, Fanger $\mathrm{H}$. Use of avidin-biotin peroxidase complex $(\mathrm{ABC})$ in immunoperoxidase techniques: a comparison between $\mathrm{ABC}$ and mislabelled antibody (PAP) procedures. J Histochem Cytochem 1981;29:577-80.

"Sternberger LA, Hardy PH Jr, Cuculis JJ, Meyer HG. The unlabelled antibody enzyme method of immunohistochemistry preparation and properties of soluble antigen-antibody complex (horseradish peroxidase-antihorseradish peroxidase) and its use in the identification of spirochaetes. $J$ Histochem Cytochem 1970;18:315.

${ }^{10}$ Cameron RG, Seemayer TA, Wang NS. Small cell malignant tumours of the thyroid gland: a light and microscopic study. Hum Pathol 1975; 6:731-40.

"Gatter KC, Alcock C, Heryet A, et al. The differential diagnosis of routinely processed anaplastic tumours using monoclonal antibodies. Am J Clin Pathol 1984;82:33-43.

12 Warnke RA, Gatter KC, Falini B. The diagnosis of human lymphoma using monoclonal antileukocyte antibodies. $N$ Engl J Med 1984;309: 1275-81.

${ }^{13}$ Sloane JP, Ormerod MG. Distribution of epithelial membrane antigen in normal and neoplastic tissues and its value in diagnostic tumour pathology Cancer 1981;47:1786-95.

14 Delsol G, Gatter KC, Stein H, et al. Human lymphoid cells express epithelial membrane antigen. Lancet 1984;ii: 1124.

is Burt A, Goudie RB. Diagnosis of primary thyroid carcinoma by immunohistological demonstration of thyroglobulin. Histopathology 1979;3:279.

Requests for reprints to: $\mathrm{Dr}$ AD Burt, University Department of Pathology, Western Infirmary, Glasgow G11 6NT, Scotland. 